\title{
Cone Beam Computertomography (CBCT) in Interventional Chest Medicine - High Feasibility for Endobronchial Realtime Navigation
}

\author{
Wolfgang Hohenforst-Schmidt ${ }^{1}$, Paul Zarogoulidis ${ }^{2}$, Thomas Vogl ${ }^{3}$, J Francis Turner ${ }^{4}$, Robert Browning ${ }^{5}$, \\ Bernd Linsmeier ${ }^{1}$, Haidong Huang, ${ }^{6}$ Qiang Li ${ }^{6}$, Kaid Darwiche7, Lutz Freitag7, Michael Simoff ${ }^{8}$, Ioannis \\ Kioumis², Konstantinos Zarogoulidis², Johannes Brachmann ${ }^{1}$ \\ 1. II Medical Department, "Coburg" Regional Hospital, University of Wuerzburg, Coburg, Germany. \\ 2. Pulmonary Department-Oncology Unit, "G. Papanikolaou" General Hospital, Aristotle University of Thessaloniki, Thessaloniki, Greece \\ 3. Department of Diagnostic and Interventional Radiology, Goethe University of Frankfurt, Frankfurt, Germany. \\ 4. Interventional Pulmonary \& Critical Care Medicine Cancer Treatment Centers of America. \\ 14200 W. Celebrate Life Way Goodyear, AZ, USA. \\ 5. Pulmonary and Critical Care Medicine, Interventional Pulmonology, National Naval Medical Center, Walter Reed Army Medical Center, Be- \\ thesda, MD, USA. \\ 6. Department of Respiratory Diseases, Changhai Hospital/First Affiliated Hospital of the Second Military Medical University, Shanghai, People's \\ Republic of China. \\ 7. Department of Interventional Pneumology, Ruhrlandklinik, West German Lung Center, University Hospital, University Duisburg-Essen, Essen, \\ Germany. \\ 8. Bronchoscopy and Interventional Pulmonology, Pulmonary and Critical Care Medicine, Henry Ford Hospital, Wayne State University, School of \\ Medicine, MI, USA.
}

$\triangle$ Corresponding author: Paul Zarogoulidis, Pulmonary Department, “G. Papanikolaou” General Hospital, Aristotle University of Thessaloniki, Thessaloniki, Greece. Fax: 00302130992433 Mobile: 00306977271974 Email: pzarog@hotmail.com.

(C) Ivyspring International Publisher. This is an open-access article distributed under the terms of the Creative Commons License (http://creativecommons.org/ licenses/by-nc-nd/3.0/). Reproduction is permitted for personal, noncommercial use, provided that the article is in whole, unmodified, and properly cited.

Received: 2013.02.14; Accepted: 2014.02.23; Published: 2014.03.09

\begin{abstract}
Introduction: Currently there are several advanced guiding techniques for pathoanatomical diagnosis of incidental solitary pulmonary nodules (iSPN): Electromagnetic navigation (EMN) with or without endobronchial ultrasound (EBUS) with miniprobe, transthoracic ultrasound (TTUS) for needle approach to the pleural wall and adjacent lung and computed tomography (CT) -guidance for (seldom if ever used) endobronchial or (common) transthoracical approach. In several situations one technique is not enough for efficient diagnosis, therefore we investigated a new diagnostic technique of endobronchial guided biopsies by a Cone Beam Computertomography (CBCT) called DynaCT (SIEMENS AG Forchheim, Germany). Method and Material: In our study 33 incidental solitary pulmonary nodules (iSPNs) (28 malignant, 5 benign; mean diameter 25 $+/-12 \mathrm{~mm}$, shortest distance to pleura $25+/-18 \mathrm{~mm}$ ) were eligible according to in- and exclusion criteria. Realtime and onsite navigation were performed according to our standard protocol.22 All iSPN were controlled with a second technique when necessary and clinical feasible in case of unspecific or unexpected histological result. In all cases common guidelines of treatment of different iSPNs were followed in a routine manner. Results: Overall navigational yield (ny) was $91 \%$ and diagnostic yield (dy) $70 \%$, dy for all accomplished malignant cases $(n=28)$ was $82 \%$. In the subgroup analysis of the invisible iSPN ( $n=12$, I I malignant, I benign; mean diameter $15+/-3 \mathrm{~mm}$ ) we found an overall dy of $75 \%$. For the first time we describe a significant difference in specifity of biopsy results in regards to the position of the forceps in the 3-dimensional volume (3DV) of the iSPN in the whole sample group. Comparing the specifity of biopsies of a 3D-uncentered but inside the outer one third of an iSPN-3DV with the specifity of biopsies of centered forceps position (meaning the inner two third of an iSPN-3DV) reveals a significant ( $\mathrm{p}=0,0375 \mathrm{McNemar}$ ) difference for the size group $(>1 \mathrm{~cm})$ of 0,9 for centered biopsies vs. 0,3 for uncentered biopsies. Therefore
\end{abstract}


only 3D-centered biopsies should be relied on especially in case of a benign result. Conclusion:The diagnostic yield of DynaCT navigation guided transbronchial biopsies (TBB) only with forceps is at least up to twofold higher than conventional TBB for iSPNs $<2 \mathrm{~cm}$. The diagnostic yield of DynaCT navigation guided forceps TBB in invisible SPNs is at least in the range of other navigation studies which were performed partly with multiple navigation tools and multiple instruments. For future diagnostic and therapeutic approaches it is so far the only onsite and realtime extrathoracic navigation approach (except for computed tomography (CT)-fluoroscopy) in the bronchoscopy suite which keeps the working channel open. The system purchase represents an important investment for hospitals but it is a multidisciplinary and multinavigational tool with possible access via bronchial airways, transthoracical or vascular approach at the same time and on the same table without the need for an expensive disposable instrument use.

Key words: solitary pulmonary nodule, cone-beam computed tomography (CBCT), electromagnetic navigation bronchoscopy (EMN, ENB), transbronchial biopsy (TBB).

\section{Introduction}

Incidence of lung cancer is currently $1 / 1850-1 / 2000$ per inhabitant in the United States which means $>200000$ newly diagnosed cases and $>160000$ deaths per year. ${ }^{1}$ The population is aging with more chronic obstructive pulmonary disease patients having one of the most important risk factors for lung cancer. ${ }^{2}$ The prevalence of iSPNs in an unbiased population on thoracic imaging with $\mathrm{X}$-ray photography (XR) is described with $0.2 \%{ }^{3}$ and with CT $0.5 \%{ }^{4}$. In comparison to a population at risk the values are different for XR 7\% and CT $23 \%$, which of these are malignant in the XR group with $0.4 \%$ and in the CT group with $2.7 \%$. From a clinical perspective this means fifty times more iSPNs in patients at risk (in comparison to a population without risk) but only every tenth is malignant. Following actual guidelines for the management of iSPN this would dramatically increase the rate of futile thoracotomy especially in a group with patients at risk e.g. poor lung function if no other pre-operative diagnostic approach (preferably with navigation support) is accomplished. ${ }^{5}$ Furthermore, the relative risk of an iSPN in an unbiased population on $\mathrm{CT}$ to develop lung cancer according to size is 6 : $<4 \mathrm{~mm} \mathrm{1 \% ;} \mathrm{>8mm} \mathrm{10-20 \% ;} \mathrm{<1cm:} 15 \% 7,8$; $>20 \mathrm{~mm}$ : $50 \%$; $>3 \mathrm{~cm}$ : $>95 \% 7,8$. Moreover volume doubling time (VDT) is not differentiating well between malignant and benign': Small cell lung cancer (SCLC) shows a typical VDT of 30 days which means from a clinical perspective an easy cut-off value. On the other hand small well differentiated adenocarcinomas of the lung (AC) appear with a VDT of 500-750 days which is a difficult-to-handle cut-off. From a clinical perspective VDT has no major value. It was also observed in the national lung screening trial (NCI U.S.) ${ }^{9}$ that using low dose CT $(<1,5 \mathrm{mSv})$ in a patient population at risk (smoking history) reduces mortality due to lung cancer around $20 \%$. The relative risk of an iSPN in an unbiased population on positron emission tomography (PET)-CT to become lung cancer ac- cording to SUV>2.5 is $90 \% .{ }^{10}$ Additionally, we have a reduction of "futile thoracotomies" for iSPN with the addition of PET-CT after conventional staging in non-small cell lung cancer (NSCLC) ${ }^{11}$ from $42 \%$ without PET-CT to $21 \%$ with PET-CT (but with no improvement of overall survival). Based on this information neither CT nor PET-CT is the definite solution for the individual question of dignity of an iSPN, once again it remains the hand of the interventional doctor to clarify the situation with the leading words of "tissue is the issue". Based on the American College of Chest Physicians (ACCP 2007) guidelines on TBB ${ }^{12}$ the expected sensitivity for all malignant iSPN $<3 \mathrm{~cm}$ is 33 to $50 \%$. From a clinical perspective this guideline is not covering the aspect of "correct negative results": At the end of a diagnostic process one wants to know if a benign histological result indicates a pathoanatomically benign iSPN. This is not the value of sensitivity but of specifity of a navigated biopsy indicating if a benign (possibly correct negative) 'navigated histology represents a truly benign iSPN. Electromagnetic navigation and fluoroscopy together show a $\mathrm{dy}$ in the range of $63-74 \%$ for iSPN with a mean diameter of at least $2,4 \mathrm{~cm}+/-1,5 \mathrm{~cm}$ or bigger. ${ }^{12} \mathrm{In}$ iSPN $<2 \mathrm{~cm}$ the application of miniprobe EBUS +/- fluoroscopy in addition to multiple instruments (sheath, curette, brush and forceps) the dy was reported to be between $44,4 \%$ up to $72.8 \%$. The combination of electromagnetic navigation and miniprobe EBUS +/multiple instruments raised the number of dy even for iSPN $<2 \mathrm{~cm}$ over $80 \% .{ }^{12}$ However the cost per examination in comparison to conventional TBB is at least $1000 €$ and therefore very expensive. Reimbursement of this technique is not available in many health systems. There are some aspects to remember: a) These navigation tools are used endobronchially and therefore block the working channel. Only Veran Systems has invented electromagnetic fitted forceps and other tools so far. Except for this system it is of notice that 
neither the performance of the all-decisive biopsy nor any endobronchial therapy (in future) can be monitored by these devices. B) The iSPNs in upper lobes are easier to manage than in lower lobes - this counts as well for all sophisticated devices. One has to remember that due to the elastic recoil all navigation systems working as 2-step-system the dataset for virtual bronchoscopy is accomplished in an awake patient taking a deep breath while the examination with this 3D-dataset takes place later in a patient asleep with a total different diaphragm position. This factor influences especially the navigation results in the lower lobes. C) Despite the application of sophisticated devices the dy in large $(>3 \mathrm{~cm})$ iSPNs is interestingly enough not higher - maybe worse than in smaller iSPNs. ${ }^{12}$ D) It has to be accepted that in general there is an obvious difference between navigational and diagnostic yield of in average of $20 \% .13$ Therefore one should try to identify what influences a successful navigation with high specifity and high sensitivity for malignancy besides size and lobar location for iSPNs. The following items should be considered:

A) The growing pattern of a "homogenous radiological" iSPN is spoken from a pathoanatomical point of view in reality inhomogenous: All bronchologists experience often the finding of benign histology like organizing pneumonia / anthracosis or scar at the rim of the radiological appearance of a malignant iSPN. Therefore in case of benign histology one has to ask if we can believe in his own results.

B) The detectability of distal airways is even in CT limited to a diameter of no less than $1,5 \mathrm{~mm}$ which is around the $8^{\text {th }}$ segmentation of the bronchial tree.

C) The site of the iSPN in relation to anatomical pathway influences the yield of a navigated endobronchially approach: If forceps/brush is used an anatomical pathway ( e.g. a bronchus sign) into the lesion is needed. If needle is used an extraanatomical pathway is possible. Notable is the fact that less steerability of a peripheral needle position only allows to advance an iSPN over $1-2 \mathrm{~cm}$ and needs greater experience than a conventional TBB.

D) Distortion of airway anatomy allows electromagnetic navigation systems the statement of "successful navigational yield" although the diagnostic yield is zero. This is due to the fact that the operator can distort by manual power via the bronchoscope the actual airway anatomy so that a bronchial airway containing the EMN-sensor only "kisses" the correct position without anatomical opening towards the target of the neighboured area.

E) Electromagnetic navigation systems always depend on at least one pre-endoscopy CT which is obligatory for the realisation of a virtual bronchoscopy dataset for the second step: The examination itself. In other words: All electromagnetic systems so far work as a 2-step-system, the 3-dimensional navigation dataset is not composed during the definite examination. Therefore these systems may give a realtime feedback of the navigation process but the anatomical 3D-datset is not composed onsite - there is always a time- and performance-gap between anatomical reality and virtual navigation anatomy.

In regards to the growing pattern of an iSPN positioning could be measured in comparison to the projection of the forceps and its position to the outlined iSPN-3DV projection. In this study we always aimed to reach the inner 2/3 of an iSPN-3DV (Figure $1)$ : This is our definition of a centered biopsy. In contrast the outer $1 / 3$ of an iSPN-3DV is still inside but uncentered and therefore of expected different value. Both situations - centered and inside - have the quality of a positive navigational yield. This concept is based on the fact that a bronchus sign leading somewhere into an iSPN-3DV is one of the strongest predictors of a correct diagnostic yield. ${ }^{14}$
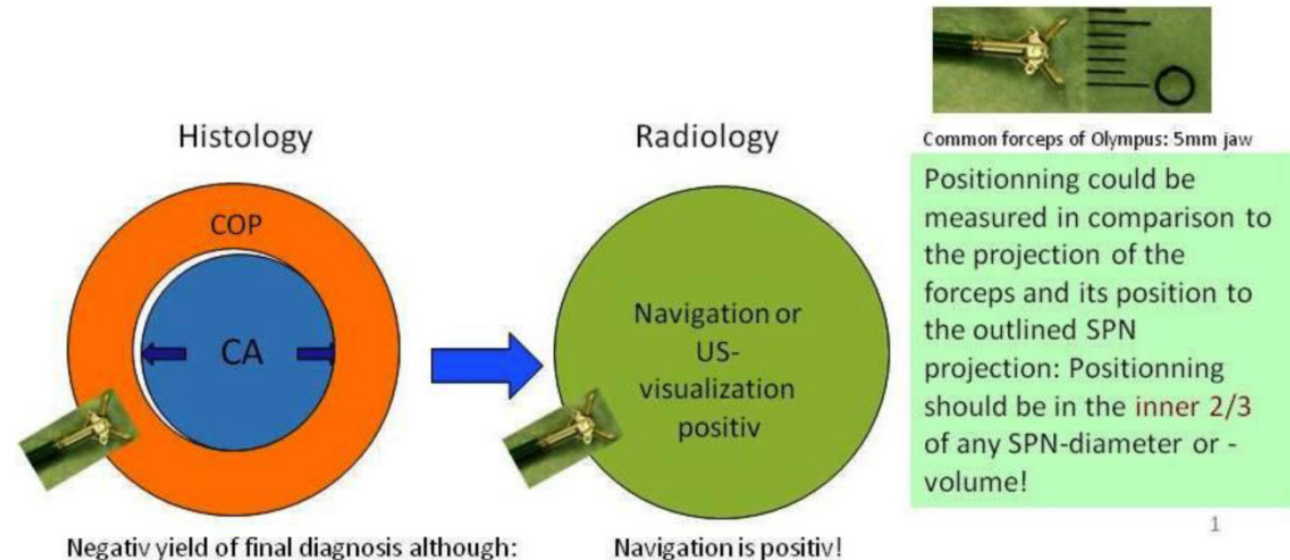

Negativ yield of final diagnosis although:

Navigation is positiv!

1

Figure I: In big SPN: Accuracy in regards to diagnosis is a question of detection AND positionning intratumoral. In regards to navigation accuracy is defined by detection only. (intratumoral $=$ inside/borderline or centered) In small SPN $<15 \mathrm{~mm}$ (per definition): Accuracy in regards to diagnosis and navigation is a question of (more or less) detection only (hit or fail). 
In regards to the detectability of small bronchial airways our standard examination protocol uses intermediate high airway pressure ${ }^{15}$ : It is expectable that the timely limited hyperinflation will deliver more CT-contrast (air in contrast to bronchial wall, parenchyma and consolidation) and dilate smaller bronchioli meaning a deeper segmentation than normal, at least in some DynaCT-datasets if any patient or organ movement could be avoided by the protocol. ${ }^{15}$ Therefore we expect partly a higher resolution with our DynaCT protocol than on conventional HRCT and therefore in principal better results.

In regards to EMN devices we already described that DynaCT guided endobronchial navigation as one-step realtime and onsite navigation has clear advantages over all 2-step-navigation methods. ${ }^{15}$ In our current study we will elicit the place of DynaCT in interventional chest medicine.

\section{Patients and Methods}

\section{General considerations on Cone-Beam Computed Tomography (CBCT)}

Digital flat-panel detector CBCT has been adapted for the use with C-arm systems. This configuration allows the standard 2D projection functionality like fluoroscopy as well as volumetric computed tomography (CT) capabilities within the interventional suite. Currently this technology is used for many fields of interventional radiology procedures like cerebral aneurysm characterization and intra-arterial liver tumor embolization. The central difference between conventional multidetector $\mathrm{CT}$ and CBCT is that CBCT acquires information using a high-resolution two-dimensional detector instead of detector slices used for Multi Slice CT (MSCT). For acquiring the $3 \mathrm{D}$ dataset the $\mathrm{C}$-arm needs to rotate by $200^{\circ}\left(180^{\circ}\right.$ plus fan angle) around the target that remains stationary during acquisition. ${ }^{16}$ For our image acquisition we used the 5 seconds run with 248 single 2D-projections. This protocol (without contrast agent except for air) is providing sufficient image quality for clearly displaying the bronchial structures as well as the target itself. These data are sent automatically to a workstation where the reconstruction is finished within less than 15 seconds (s). At the workstation the target itself as well as the path within the bronchial tree are identified and marked with manual annotation tools (Siemens iguide toolbox). In the following step these markers are projected and overlaid to the fluoroscopic image. This technique allows the physician to match his instruments (bronchoscope, forceps) to the overlay and in consequence navigate correctly to the (fluoroscopy-invisible) target. The major limitation of this technique is a significant lower image quality if there is target movement during 3D acquisition. For eliminating this factor the absence of spontaneous respiration is mandatory - the patient is put into apnea. The connected ventilation system is maintaining the inspiration phase during acquisition as well as the navigation process. This leads to an accurate match between the 3D dataset and the fluoroscopic image. For visualization of lung structures there is no need for artificial contrast medium except for air which makes 800-1000 HU contrast difference in comparison to bronchial wall. The issue of getting the contrast medium AIR into small subpleural bronchioli can be resolved with the following rules. (Figure 4.). The diaphragm should be kept still, no movement should be performed especially during data acquisition. The small subpleural bronchioli and especially the $>8^{\text {th }}$ generation should be dilated for CT-contrast enhancement. This action can be performed with supranormal constant airway pressure. Moreover the patient should be kept in the same position for data acquisition and examination. Complete and constant apnea by distinct sedation protocol during acquisition have been applied up to 90 minutes without any adverse effects. ${ }^{15}$ Complete apnea and constant inspiratory positioning of the diaphragm should be acquired between acquisition and navigation to realize best biopsy results. ${ }^{17}$ (Figure 5.). Furthermore a distinct jet-ventilation protocol should be applied with nasal-jet-catheter to keep optimal ventilation setting and periods of complete apnea altering with sufficient ventilation for biopsy under expiration (in order to reduce the risk of pneumothorax). ${ }^{15}$ (Figure 6,7,8.).

\section{Study design}

Prospective real world, all comers study design including forty-nine patients with 50 iSPNs. There was no limit in regards to age (24-89 years), body mass index (mean $31 \mathrm{~kg} / \mathrm{m} 2$ height, maximum $40 \mathrm{~kg} / \mathrm{m} 2$ height) or clinical condition of any comorbidities ( any stage of COPD, heart insufficiency, etc.).

\section{Purpose}

The purpose of our study was to investigate the accuracy of DynaCT navigation as an onsite and extrathoracical navigation tool in our bronchoscopy suite especially in comparison to standard transbronchial biopsy (TBB) under fluoroscopy. Therefore we did not use any other instrument for obtaining tissue than forceps for TBB. A special focus was put on the so called "invisible" iSPN on fluoroscopy (e.g. $<2 \mathrm{~cm}$ depending on the location in the lung) and the information given by the iSPN-3DV-dataset. The visibility was announced by one blinded nurse before DynaCT under fluoroscopy in the DynaCT suite. By 
choice of the physician it was allowed to use peripheral navigation devices like Superdimension`s steering catheter (without the electromagnetic device) or curette of Olympus.

\section{Inclusion and Exclusion Criteria}

1. The patients were included only after informed consent (especially over additional radiation exposure by DynaCT application and intermediate high pressure sustained jet-ventilation). All patients were presented with at least one iSPN on initial (external) XR or thoracic CT (TCT) scan. All patients were part of the daily routine program in our hospital.

2. Before enrollment all patients had to prove favorable conditions for endoscopic biopsies under jet-catheter-ventilation in the DynaCT suite and no major ear, nose and throat (ENT) problems. At all patients without an initial TCT but with suspicious iSPN on XR we performed after enrollment an additional diagnostic standard TCT. Before DynaCT navigation all enrolled patients had an additional transthoracic ultrasound to exclude pleural effusion in the DynaCT suite due to the fact that compression of distal airways (DA) by pleural effusion would influence negatively the visualization of DA hyperinflation and increase the risk of pneumothorax by reduced compliance.

3. In standard TCT projections the largest diameter were measured for height, width and depth (h,w and d). All diameters had to be $\geq 10 \mathrm{~mm}$ at least in the lung window on initial TCT.

4. If an alternative way of diagnosing iSPN than TBB were feasible (e.g. transthoracic puncture in case of a pleural carcinosis/exudates, ultrasound -guided transthoracic needle approach in case of subpleural consolidation, central biopsy in case of endoscopic central process) the patient would automatically be excluded from the DynaCT navigation analysis.

5 . The inability to visualize a peripheral bronchial airway towards the iSPN (e.g. extreme distortion) after the segmental ostia until $1 \mathrm{~cm}$ towards the target lead to exclusion from the DynaCT navigation analysis.

6. Pleural effusion lead to exclusion of the analysis as well as diaphragmatic paresis.

7. iSPN highly suspicious for hemartoma were not enrolled for biopsy and only followed for 9 months. This is due to the fact that the establishment of a diagnosis of a hemartoma is rarely successful with a histology given by forceps.

8. Any other iSPN was controlled by a second method like surgery, transthoracic approach, and if feasible only follow-up (3,6 and 9 months) in case of benign histology.

\section{Patients}

In total forty-nine patients were enrolled with fifty iSPNs, exclusion of seventeen iSPN were documented, therefore thirty-three iSPNs to be analyzed for DynaCT guided endobronchial navigation forceps biopsy. In detail we have found on the 17 initially excluded iSPNs the following additional findings:

a) Pleural carcinosis (one case) and contact to parietal pleura (7cases: 3 benign, 4 malignant).

b) One iSPN < 10mm in at least one diameter: In follow-up over 9 months this case was diagnosed as a benign nodule.

c) Due to diaphragmatic paresis we excluded one iSPN of less than $2 \mathrm{~cm}$ of the analysis which turned out to be malignant.

d) 2 initially enrolled iSPNs on XR fulfilled the criteria of a hemartoma on TCT and were followed up for 9 months without any change in appearance.

e) We have found 3 incidental central carcinomas.

f) One case appeared with an iSPN of $15 \mathrm{~mm}$ only at interlobar site belonging more to the pleural surface without bronchial access. This nodule was diagnosed as adenocarcinoma of the lung by surgery.

g) There were 2 missed follow-ups due to early unexpected cardiovascular death and denial of surgery including disappearance of the patient. Both cases presented with a negative navigational yield. Both cases are believed to be malignant.

All these diagnosis were made upon conventional techniques without the use of a CBCT.

\section{Feasibility}

In total no life threatening major adverse effects were observed despite intermediate apnea and hyperinflation for navigation. There were two pneumothoraces treated by drainage in the next day $(2 / 33$ means $\leq 6 \%$ ). One non-life threatening bradycardia and hypotension occurred due to aggressive sedation (midazolam, propofol and remifentanyl) which was treated with saline, atropine and akrinor. Dry mucosa was regularly observed due to jet-ventilation without humidification. The heavier the patients the more prolonged hyperinflation pressure by jet-catheter ventilation was needed (working pressure up to 3.5 bar for 20 seconds controlled and uptitrated stepwise under fluoroscopy looking at the deepest position of the diaphragm). Moreover, all examinations were performed in flexible technique with nasal jet-catheter. A rigid bronchoscopy was available but it was reducing the 3D-dataset quality by metal artifacts. With the hereby used protocol there was no essential need for anaesthesiologists.$^{15}$ 


\section{General consideration}

\section{Navigation is positive if}

In posterior-anterior (pa) standard projection the targeted volume can be reached inside the borders by the forceps and the 3-edges-angulation is positive keeping the forceps "inside the projection of the targeted volume". Inside the borders means as forceps positioning: Position of the forceps is in the outer $1 / 3$ of an iSPN-3DV. Centered means: Position of the forceps is in the inner $2 / 3$ of an iSPN-3DV.

\section{3-edges-angulation}

The degree needed for angulation to accurate spatial accordance between anatomy and navigation is dependent upon the nodule size and position of the forceps/instrument. In general an angulation of 20-30 degrees at least is needed according to other DynaCT applications. A positive 3-edge-angulations means a successful navigation and consists of a positive pa-projection and 3 different angulations in different directions "over the edges". The technique of 3-edge-angulation is described elsewhere. ${ }^{15}$

\section{Results}

Out of thirty-three iSPNs twenty-eight iSPNs were diagnosed to be malignant and five were benign. In specific we missed one benign and two malignant iSPNs due to negative navigation. In 2 patients follow-up method was not possible due to denial of surgery and death. The overall ny was $91 \%$ in the whole group. The overall dy was $70 \%$. The dy for all possible malignant iSPNs was $82 \%$. If we include the two patients assuming malignancies which were excluded for missed follow-ups (due to death/denial for surgery) the dy for malignancy was $77 \%$ in the whole sample. (Figure 2.) For all iSPN $>1 \mathrm{~cm}$ the McNemar-Test $(p=0.0375)$ for specifity inside vs. centered biopsies showed a significant result with high differences: The specifity of centered biopsies was $0,67-1,0$ overall subgroups according to size, for inside biopsies 0,29-0,33. (Figure 3.)

Looking at the size subgroups $(\leq 2 \mathrm{~cm}$ and $>2 \mathrm{~cm}$ ) overall diagnostic yield was $75 \%(15 \pm 3 \mathrm{~mm})$ and $67 \%$ $(30 \pm 11 \mathrm{~mm})$ respectively. In both subgroups the sensitivity for malignancy was $82 \%$.

\begin{tabular}{|c|c|c|c|c|c|c|c|c|c|c|}
\hline isPN numbers & $\mathrm{N}=33$ & & $\mathrm{~N}=23$ & & $\mathrm{~N}=10$ & & $\mathrm{~N}=12$ & & $\mathrm{~N}=21$ & \\
\hline diam.+dist. in $\mathrm{mm}$ : & all $d .>1 \mathrm{~cm}$ & $25 / 26 / 24 ; 25$ & all d. $1>\leq 3 \mathrm{~cm}$ & $18 / 20 / 19 ; 25$ & one $\mathrm{d} .>3 \mathrm{~cm}$ & $41 / 39 / 36 ; 23$ & all d.1 $>-\leq 2 \mathrm{~cm}$ & $14 / 15 / 15 ; 29$ & one d. $>2 \mathrm{~cm}$ & $31 / 31 / 29 ; 21$ \\
\hline std. dev. in mm: & & $13 / 12 / 10 ; 18$ & & $5 / 5 / 5 ; 17$ & & $10 / 12 / 5 ; 19$ & & $3 / 3 / 2 ; 17$ & & $12 / 11 / 8 ; 17$ \\
\hline & Histology & & Histology & & Histologie & & Histology & & Histology & \\
\hline Navigation & positive & negative & positive & negative & positiv & negativ & positive & negative & positive & negative \\
\hline inner $2 / 3$ & 22 & 1 & 16 & 1 & 6 & 0 & 9 & 1 & 13 & 0 \\
\hline outer $1 / 3$ & 1 & 6 & 0 & 4 & 1 & 2 & 0 & 1 & 1 & 5 \\
\hline negativ & 0 & 3 & 0 & 2 & 0 & 1 & 0 & 1 & 0 & 2 \\
\hline & all $d .>1 \mathrm{~cm}$ & & all d. $1>-\leq 2 \mathrm{~cm}$ & & all d. $1>\leq 3 \mathrm{~cm}$ & & lone d. $>2 \mathrm{~cm}$ & & one $d .>3 \mathrm{~cm}$ : & \\
\hline & CENTERED & INSIDE & CENTERED & INSIDE & CENTERED & INSIDE & CENTERED & INSIDE & CENTERED & INSIDE \\
\hline sensitivity & 0,96 & 1,00 & 1,00 & 1,00 & 1,00 & 1,00 & 0,93 & 1,00 & 0,86 & 1,00 \\
\hline specifity & 0,90 & 0,30 & 0,67 & 0,33 & 0,86 & 0,29 & 1,00 & 0,29 & 1,00 & 0,33 \\
\hline PPV & 0,96 & 0,77 & 0,90 & 0,82 & 0,94 & 0,76 & 1,00 & 0,74 & 1,00 & 0,78 \\
\hline NPV & 0,90 & 1,00 & 1,00 & 1,00 & 1,00 & 1,00 & 0,88 & 1,00 & 0,75 & 1,00 \\
\hline \begin{tabular}{|l} 
accuracy \\
\end{tabular} & 0,94 & 0,79 & 0,92 & 0,83 & 0,96 & 0,78 & 0,95 & 0,76 & 0,90 & 0,80 \\
\hline diagnostic yield & 0,70 & 0,70 & 0,75 & 0,75 & 0,70 & 0,70 & 0,67 & 0,67 & 0,70 & 0,70 \\
\hline
\end{tabular}

Figure 2. Statistics along with navigation. 


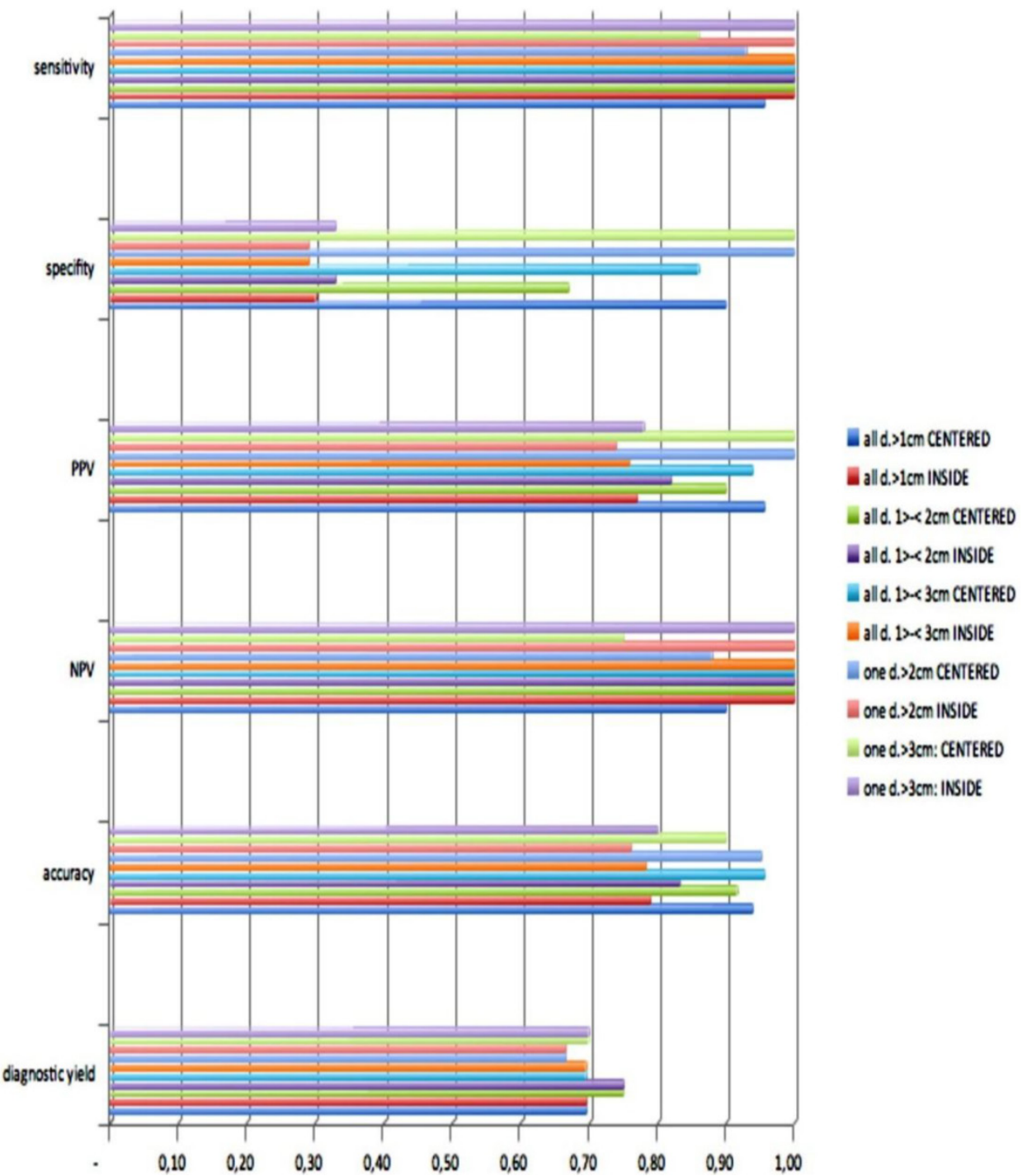

Figure 3. Statistical analysis.

\section{DynaCT-rotation $=$ dataset acquisition \\ DynaCT $=\mathrm{CT}+$ fluoroscopy in ONE \\ open suite for bronchoscopy/surgery}
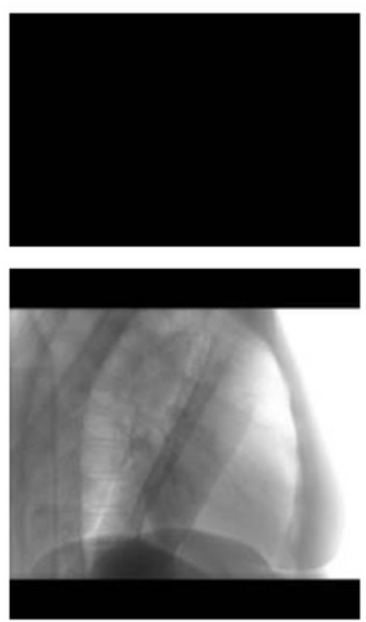

Post-Processing of 3-dimensional dataset

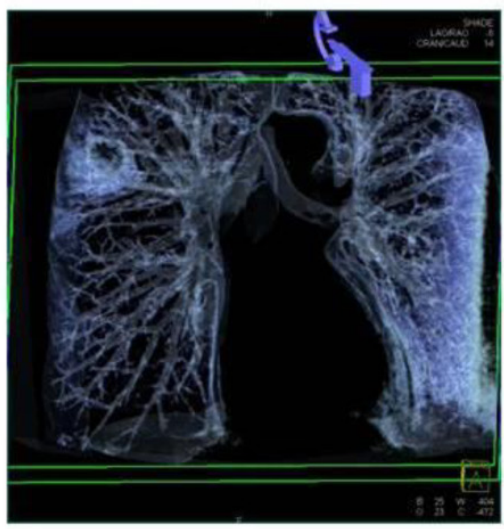

Figure 4. Where is the place for DynaCT in Interventional Chest Medicine? 


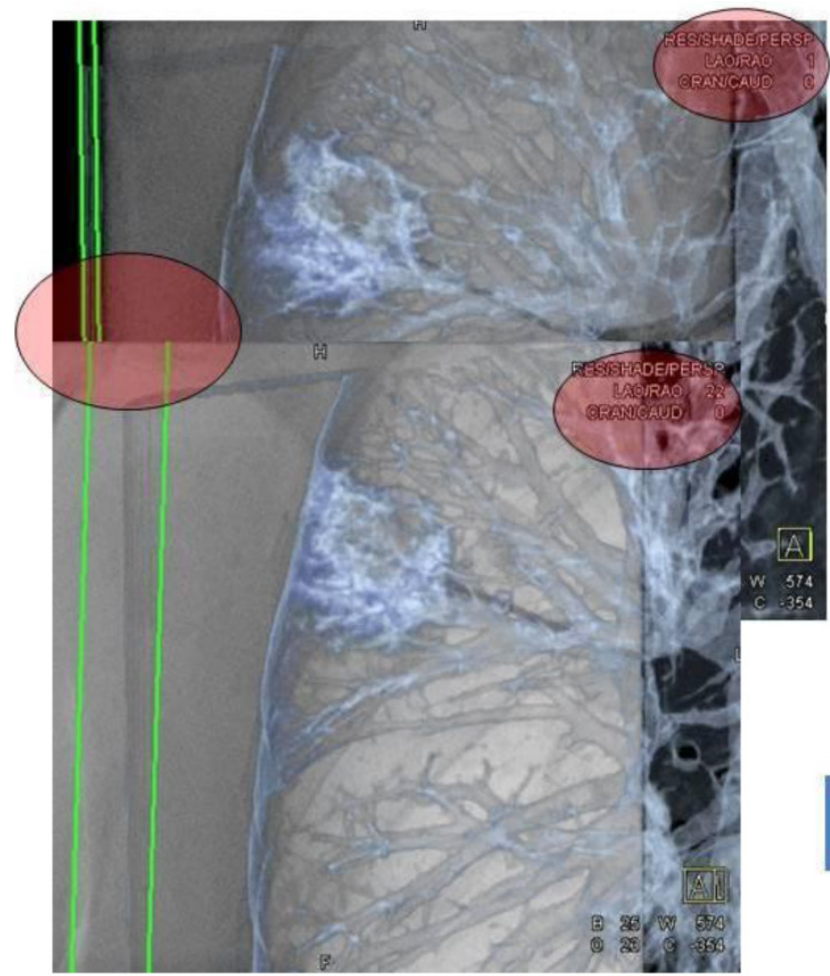

Real-time overlay during bronchoscopy: Patient not deeply sedated and still coughing.

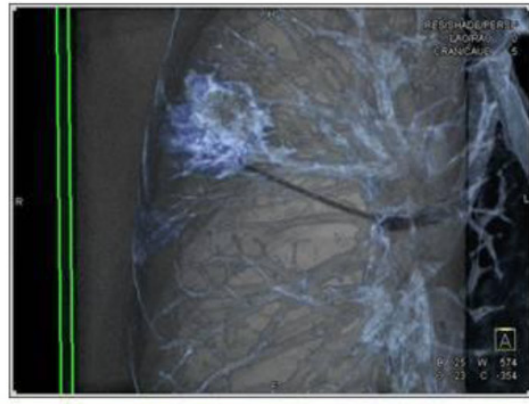

Segmentation up to the 17 th generaton (max.)

Figure 5. CT-dataset in XR: 3-dimensional volume information projected into c-arm fluoroscopy.
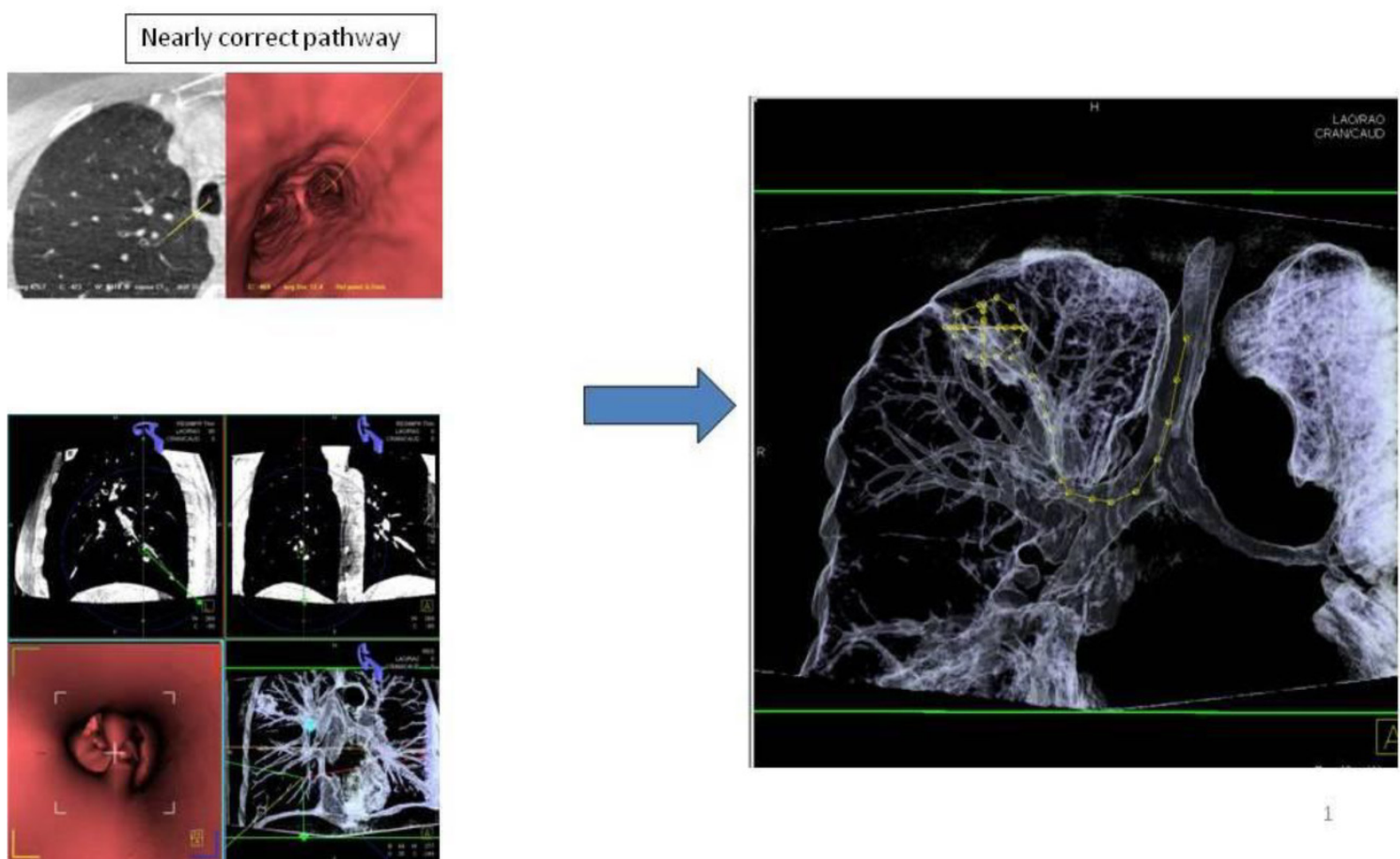

Figure 6. Applying 3-dimensional DynaCT dataset: 'Dotting' a pathway in virtual endoscopy or standard CT-projections towards the target up to the I2th segmentation. 

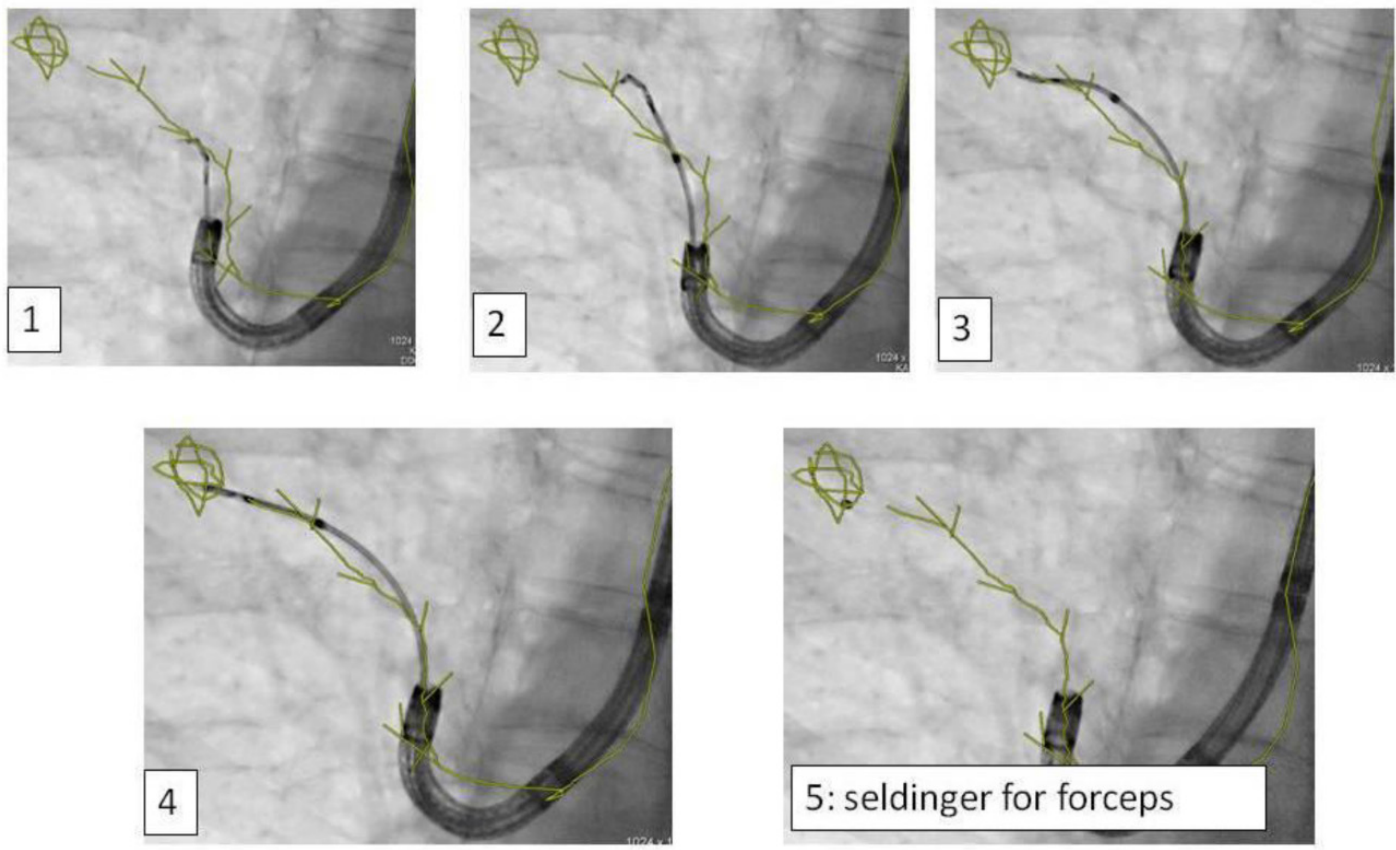

Figure 7. Advancing an iSPN by a navigation device along the 3D-overlay: Distortion of the airway due to navigation force.

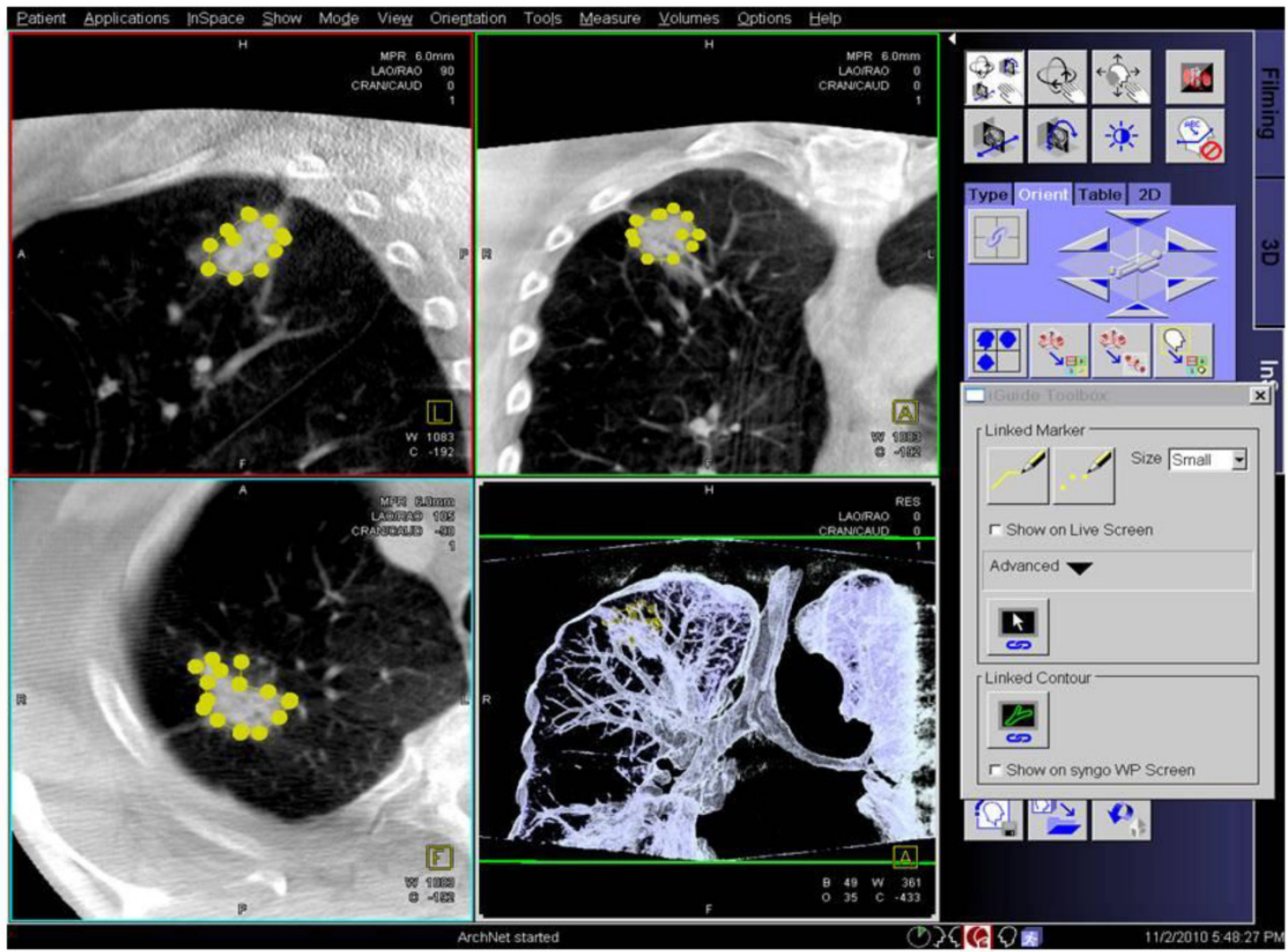

Figure 8. Applying 3-dimensional DynaCT-dataset: Outlining the target in 3 standard axis.

\section{Discussion}

Comparing DynaCT with fluoroscopy guided TBB of iSPNs the following results where found: Sensitivity for malignancy in this study for iSPN $\leq 2 \mathrm{~cm}$ $(15 \mathrm{~mm} \pm 3 \mathrm{~mm})$ was $82 \%$ which is much better than the described sensitivity for fluoroscopy guided con- ventional TBBs in iSPNs of the same size with a mean value of $33 \%$. ${ }^{17-19}$ The same counts for the described sensitivity of malignant iSPNs $>2 \mathrm{~cm}$ with a mean value of $62 \%$ in comparison to again $82 \%$ in this study. ${ }^{17-19}$ Comparing DynaCT guided TBBs in regards to invisibility of iSPNs (that is to say the sub- 
group of $\leq 2 \mathrm{~cm}$ with a mean diameter of $15 \pm-3 \mathrm{~mm}$ ) we found a sensitivity for malignancy of $82 \%$ and for the size subgroup of $\leq 3 \mathrm{~cm}$ (with a mean diameter $19+/-5 \mathrm{~mm}$ meaning nearly invisibility) a sensitivity of $76 \%$. This is comparable to published literature from Eberhardt and colleagues showing a sensitivity of $70,3 \%$ for invisible iSPNs diagnosed with the help of minprobe EBUS under fluoroscopy. ${ }^{14}$ In the case where the iSPN cannot be reached intrabronchially, the iguide needle guidance for transthoracic approach should be used. ${ }^{20,21}$ Comparable accuracy between the DynaCT guided transthoracic approach ${ }^{21}$ (93\%) and the DynaCT guided endobronchial approach in centered biopsies (94\%) has been observed in our study. Of notice is the fact that in our study only in $6 \%$ a pneumothorax occured vs. $14 \%$ resp. $26 \%$ in the radiological study. ${ }^{21}$ Of interest is the analysis about specifity of a biopsy result in regards to the position of the forceps in the iSPN: Our results indicate that one only can trust a benign biopsy histology if it is taken of the central part (inner 2/3 of a 3DV) of the nodule. Although this is a routine and major clinical question it is not addressed to the best of our knowledge in other studies or guidelines in the area of interventional pulmonology before. And there is no other navigation instrument than CBCT (except for CT-fluoroscopy) which can 'foresee' the value of a possible biopsy along the 3D-dataset. All EMN-systems are using an artificial 'rounded' or 'smoothed' 3DV of the targeted iSPN. Our results here refer back to the 'exactly outlined' 3DV of an iSPN. In that context CBCT technology is unique.

Finally this study shows that a new method for endobronchial navigation has its limit:

A) Nearly one third of all initially enrolled iSPNs were excluded from analysis due to protocol which means a certain bias. These cases were diagnosed along the routine program of our clinic. Although the remaining number to be analyzed is small it is quite comparable with other studies. ${ }^{12}$

B) On first sight the additional radiation exposure of less than $2 \mathrm{mSv}^{15}$ is a disadvantage. Of notice is the fact that for electromagnetic navigation sytems a complete additional multi-slice TCT with an average value of around $7 \mathrm{mSv}$ is necessary ${ }^{15}$, in some systems (like Veran Systems) even 2 (in inspiration and exspiration) TCTs are required before application of such a navigation system. Therefore as shown in another publication the added radiation exposure by DynaCT navigation to a patient is in comparison to many other CT and navigation applications considerably very low. ${ }^{15}$ C) As prolonged and controlled apnea in hyperinflation is crucious for the whole method one has to be accustomed to jet-catheter-ventilation and this herein applied pro- tocol. ${ }^{15}$ D) Increased overweight or thoracical deformation limits the jet-ventilation-effect and increases the risk of pneumothorax especially over conventional TBB.

\section{Conclusion}

DynaCT guided endobronchial navigation is to the best of our knowledge (except for CT-fluoroscopy) the only realtime and onsite extrathoracic navigation system. It is therefore assumable that in future therapeutic approaches in local lung diseases under navigation guidance will take place in such a DynaCT suite as the working channel of a bronchoscope is not blocked by navigational instruments. So far there is no need for special and expensive one-way instruments. Furthermore it has the advantage if necessary to skip over to a navigated transthoracical approach on the same table during the same examination. Diagnostic yield of DynaCT guided forceps biopsies are basically twofold better than conventional guided biopsies under fluoroscopy especially in nodules less than 2 $\mathrm{cm}$. In under fluoroscopy invisible nodules the diagnostic yield of DynaCT guided TBBs are in the range of TBBs guided by other navigational tools like electromagnetic navigation systems and/or miniprobe EBUS. The purchase of such a system represents a major investment but it is at the same time a multidisciplinary tool which can be used by many other disciplines.

\section{Conflict of Interest}

WHS and JB receive honorary for studies with SIEMENS.

\section{References}

1. Jemal A, Siegel R, Ward E, Murray T, Xu J, Thun MJ. Cancer statistics, 2007. CA Cancer J Clin. 2007;57(1):43-66.

2. Kushi LH, Byers T, Doyle C, et al. American Cancer Society Guidelines on Nutrition and Physical Activity for cancer prevention: reducing the risk of cancer with healthy food choices and physical activity. CA Cancer J Clin. 2006;56(5):254-281.

3. Comstock GW, Vaughan RH, Montgomery G. Outcome of solitary pulmonary nodules discovered in an x-ray screening program. $N$ Engl J Med. 1956;254(22):1018-1022.

4. MacMahon H, Austin JH, Gamsu G, et al. Guidelines for management of small pulmonary nodules detected on CT scans: a statement from the Fleischner Society. Radiology. 2005;237(2):395-400.

5. Goeckenjan G, Sitter H, Thomas M, et al. [Prevention, diagnosis, therapy, and follow-up of lung cancer. Interdisciplinary guideline of the German Respiratory Society and the German Cancer Society--abridged version]. Pneumologie. 2011;65(8):e51-75.

6. Henschke CI, McCauley DI, Yankelevitz DF, et al. Early Lung Cancer Action Project: overall design and findings from baseline screening. Lancet. 1999;354(9173):99-105.

7. Zwirewich CV, Vedal S, Miller RR, Muller NL. Solitary pulmonary nodule: high-resolution $\mathrm{CT}$ and radiologic-pathologic correlation. Radiology. 1991;179(2):469-476.

8. Nathan MH, Collins VP, Adams RA. Differentiation of benign and malignant pulmonary nodules by growth rate. Radiology. 1962;79:221-232.

9. Aberle DR, Berg CD, Black WC, et al. The National Lung Screening Trial: overview and study design. Radiology. 2011;258(1):243-253.

10. Paesmans $\mathrm{M}$, Berghmans $\mathrm{T}$, Dusart $\mathrm{M}$, et al. Primary tumor standardized uptake value measured on fluorodeoxyglucose positron emission tomography is of prognostic value for survival in non-small cell lung cancer: update of a systematic review and meta-analysis by the European Lung Cancer Working 
Party for the International Association for the Study of Lung Cancer Staging Project. J Thorac Oncol. 2010;5(5):612-619.

11. Fischer B, Lassen U, Mortensen J, et al. Preoperative staging of lung cancer with combined PET-CT. N Engl J Med. 2009;361(1):32-39.

12. Eberhardt R, Anantham D, Ernst A, Feller-Kopman D, Herth F. Multimodality bronchoscopic diagnosis of peripheral lung lesions: a randomized controlled trial. Am J Respir Crit Care Med. 2007;176(1):36-41.

13. Huang CT, Ho CC, Tsai YJ, Yu CJ, Yang PC. Factors influencing visibility and diagnostic yield of transbronchial biopsy using endobronchial ultrasound in peripheral pulmonary lesions. Respirology. 2009;14(6):859-864.

14. Bilaceroglu S, Kumcuoglu Z, Alper H, et al. CT bronchus sign-guided bronchoscopic multiple diagnostic procedures in carcinomatous solitary pulmonary nodules and masses. Respiration. 1998;65(1):49-55.

15. Hohenforst-Schmidt W, Banckwitz R., Zarogoulidis P, Vogl T, Darwiche K, Goldberg E, Huang H, Simoff M, Li Q, Browning R., Freitag L, Turner JF, Le Pivert P, Yarmus L, Zarogoulidis K, Brachmann J. Radiation Exposure of Patients by Cone Beam CT during Endobronchial Navigation - A Phantom Study. Journal of Cancer. 2014;5(3):192-202.

16. Orth RC, Wallace MJ, Kuo MD. C-arm cone-beam CT: general principles and technical considerations for use in interventional radiology. I Vasc Interv Radiol. 2008;19(6):814-820.

17. Schreiber G, McCrory DC. Performance characteristics of different modalities for diagnosis of suspected lung cancer: summary of published evidence. Chest. 2003;123(1 Suppl):115S-128S

18. Shure D. Transbronchial biopsy and needle aspiration. Chest. 1989;95(5):1130-1138.

19. Shure D. Transbronchial needle aspiration--current status. Mayo Clin Proc. 1989;64(2):251-254.

20. Xin-Wei H, De-chao J, Gang W, Ji F, Ming-ti F. The initial application of C-arm computed tomography guided percutaneous biopsy of lung lesions. Vol poster C-1137. ECR 20122012.

21. Cheung JY, Kim Y, Shim SS, Lim SM. Combined fluoroscopy- and CT-guided transthoracic needle biopsy using a C-arm cone-beam CT system: comparison with fluoroscopy-guided biopsy. Korean J Radiol. 2011;12(1):89-96. 